\title{
The antibiotic prescription habits of consultants on post-radiotherapy patients
}

A survey of antibiotic prescribing by maxillofacial consultants for dental extractions following radiotherapy to the oral cavity A. N. Kanatas, S. N. Rogers and M. V. Martin Br Dent J 2002; 192: 157-160

Objectives To analyse the antibiotic prescribing trends for exodontia and minor oral surgery in patients with a history of radiotherapy. Also, to evaluate the use of hyperbaric oxygen as a separate modality in the prevention of osteoradionecrosis.

Method The heads of the departments at each maxillofacial unit in the UK were sent a closed-response questionnaire (total 109). Antibiotic prescribing was assessed in three case scenarios. Case one referred to a patient that had surgery alone as part of the management of head and neck cancer, and needed the surgical removal of second molar roots. Case two referred to a patient that had surgery and adjuvant radiotherapy as part of the management of head and neck cancer, and needed the same procedure as in case one. Case three included a patient with a history of surgery and adjuvant radiotherapy, who required the extraction of three mobile and periodontally-involved lower incisors. Consultants were also asked about the use of hyperbaric oxygen in the patients who had radiotherapy as part of their treatment strategy.

Results The response rate to the questionnaire was 73\%. For case one, $20 \%$ of the surgeons advocated pre-operative antibiotics for the surgical removal of lower posterior teeth. In contrast, in case two, 86\% supported pre-operative antimicrobial use for the surgical removal of the same teeth. In the third case, $63 \%$ of the clinicians supported pre-extraction antibiotics. Post-operative antibiotics were advocated by 52\% in the first case, $89 \%$ in the second case and $71 \%$ in the third case. $34 \%$ of the clinicians advocated hyperbaric oxygen for surgical removal of posterior teeth and 15\% for the extraction of mobile anterior teeth.

Conclusions The use of peri-extraction antibiotics was favoured among oral and maxillofacial surgeons. There was little enthusiasm towards the use of hyperbaric oxygen as a prophylactic measure, alone or in conjunction with an antimicrobial regime. Control randomised trials that will measure the effectiveness of hyperbaric oxygen and the appropriate use of antibiotics for prophylaxis in pre- and post-operative regimes are necessary to evaluate the use of these modalities.

\section{IN BRIEF}

- There is a lack of a totally unified approach in the management of patients with a history of radiotherapy for head and neck malignancy requiring dental extractions.

- Hyperbaric oxygen is a modality that needs to be explored as a prophylactic measure, in the patients with previous radiotherapy in need for dental extractions or minor oral surgical procedures, for its effectiveness to be fully known.

- There is clear evidence of antibiotic oversubscribing. Oral and maxillofacial surgeons would prescribe antibiotics irrespective of the risk for subsequent osteoradionecrosis.

\section{COMMENT}

This paper from a team in Liverpool reports the results of a survey concerning the use of antibiotics by British oral and maxillofacial surgeons during exodontia and minor oral surgery in patients who have had treated head and neck cancer. The primary objective of the paper was to ascertain the use of antibiotics in the prevention of osteoradionecrosis (ORN) following dentoalveolar surgery in the irradiated oral cavity. However, the study also contains important data related to the use of antibiotics in patients not at risk of this serious complication.

The results clearly show a lack of consensus in the use of antibiotics by a selected group of consultants. Although the choice of antibiotic showed some variation the 'favourites' (amoxicillin and metronidazole) were the same as those most commonly prescribed by British general dental practitioners. ${ }^{1}$ The study reports a lack of unanimity concerning the timing and duration of the antibiotic course. The fact that more consultants recommended the use of antibiotics post-operatively compared with preoperatively for dentoalveolar surgery in patients not at risk of ORN is surprising. In order to protect a wound from infection antibiotics, if indicated, should be used prophylactically. ${ }^{2}$ In view of the recommendations of the Standing Medical Advisory Committee ${ }^{3}$ concerning the optimum use of antibiotics to reduce the accumulation of new strains of resistant organisms the findings of this study are important.

The authors mention the use of hyperbaric oxygen in the prevention of ORN. Around one-third of the consultants surveyed advocated the use of hyperbaric oxygen as a preventative measure against ORN in irradiated patients having dento-alveolar surgery. Unfortunately this useful modality is not available in all centres.

The authors conclusion that randomised studies to assess the most effective antibiotic regimen to prevent osteoradionecrosis is justified from their survey. Their advice, that patients who are at risk of osteoradionecrosis should have exodontia in specialist centres, should be followed.

In an earlier study from Liverpool ${ }^{1}$ the authors concluded that guidelines and educational initiatives were required 'to encourage the rational and appropriate use of antibiotics in National Health Service general dental practice.' The results of the present study suggest that the population requiring such guidance may be larger than the general dental practitioner community.

John G. Meechan, Senior Lecturer and Honorary Consultant in Oral Surgery, University of Newcastle upon Tyne

1. Palmer N A O, Pealing R, Ireland R S, Martin M V. A study of therapeutic antibiotic prescribing in National Health Service general dental practice in England. BrDent J 2000; 188: 554-558.

2. Peterson L I. Antibiotic prophylaxis against wound infections in oral and maxillofacial surgery. J Oral Maxillofac Surg. 1990; 48: 6 17-620.

3. Standing Medical Advisory Committee (Sub-Group on Antimicrobial Resistance). The Path of Least Resistance. London: Department of Health, 1998. 